INPLASY PROTOCOL

To cite: Ren et al. The efficacy and safety of Chinese massage in the treatment of acute mastitis: A protocol for systematic review and metaanalysis of randomized controlled trials. Inplasy protocol 2021120019. doi: 10.37766/inplasy2021.12.0019

Received: 03 December 2021

Published: 03 December 2021

Corresponding author: Longsheng Ren

2633217818@qq.com

Author Affiliation:

Shandong University of

Traditional Chinese Medicine, Jinan, Shandong, China.

Support: Provincial Project

Funding.

Review Stage at time of this submission: Preliminary searches.

Conflicts of interest:

None declared.

\section{The efficacy and safety of Chinese massage in the treatment of acute mastitis: A protocol for systematic review and meta-analysis of randomized controlled trials}

Ren, X1; Guo, R²; Lv, C3; Zhao, D4; Dong, Z5; He, Z6; Wang, Q7.

Review question / Objective: The incidence of acute mastitis (AM) in lactating women has been increasing year by year. If there is no timely and appropriate treatment, AM may develop into mammary abscess and septicemia. This special situation has aroused social attention. Chinese massage has been widely used in the treatment of AM in recent years, but there is no systematic review of the effect of Chinese massage on AM. We plan to explore the efficacy and safety of Chinese massage in the treatment of AM.

Condition being studied: Chinese massage plays a role in dredging meridians, promoting qi and blood circulation, and enhancing the body's resistance to disease . In recent years, it has been reported that the effect of Chinese massage on acute mastitis is exact. This study will evaluate the effectiveness and safety of Chinese massage in the treatment of AM by using meta-analysis, aiming to provide more evidence-based evidence for the clinical treatment of AM.

INPLASY registration number: This protocol was registered with the International Platform of Registered Systematic Review and Meta-Analysis Protocols (INPLASY) on 03 December 2021 and was last updated on 03 December 2021 (registration number INPLASY2021120019).

\section{INTRODUCTION}

Review question / Objective: The incidence of acute mastitis (AM) in lactating women has been increasing year by year. If there is no timely and appropriate treatment, AM may develop into mammary abscess and septicemia. This special situation has aroused social attention. Chinese massage has been widely used in the treatment of AM in recent years, but there is no systematic review of the effect of Chinese 
massage on AM. We plan to explore the efficacy and safety of Chinese massage in the treatment of AM.

Condition being studied: Chinese massage plays a role in dredging meridians, promoting qi and blood circulation, and enhancing the body's resistance to disease . In recent years, it has been reported that the effect of Chinese massage on acute mastitis is exact . This study will evaluate the effectiveness and safety of Chinese massage in the treatment of AM by using meta-analysis, aiming to provide more evidence-based evidence for the clinical treatment of AM.

\section{METHODS}

Participant or population: Patients meeting the diagnostic criteria for AM will be selected for our study. There are no restrictions on source, nationality, race, age, etc.

Intervention: The experimental group was treated with Tuina, It also includes other similar Tuina interventions such as massage, manipulation, Chinese medicine massage, Chinese massage, and so on.

Comparator: The control group only received conventional treatment of Western medicine.

Study designs to be included: Randomized controlled trial.

Eligibility criteria: All of the studies included were RCTs of Chinese massage therapy for AM . All studies involving case reports, animal studies, reviews, basic research, and non-RCTs will be excluded.Patients meeting the diagnostic criteria for AM will be selected for our study. There are no restrictions on source, nationality, race, age, etc.

Information sources: Web of Science, Cochrane, Embase, Sinomed, China National Knowledge Infrastructure, Chongqing VIP Information, WanFang Data) on November 30, 2021. Randomized controlled trials (RCT) of Chinese massage therapy for AM were screened.

Main outcome(s): Primary outcome measure: overall clinical response rate, breast pain score.

Additional outcome(s): Secondary outcome measures: milk secretion, fever, mass size and time to resolution, White blood cell count, C-reactive protein, and the incidence of adverse reactions.

Quality assessment / Risk of bias analysis: According to the inclusion criteria and exclusion criteria, the included literature will be independently evaluated by two researchers $u$ sing the RCT bias risk assessment tool in the Cochrane evaluation manual Handbook5.4, and metaanalysis will be performed by RevMan5.4 software. Funnel plots were used to analyze whether the study had publication bias. Stata 15.0 software is used for data processing.

Strategy of data synthesis: Meta-analysis of the data from the included studies will be performed by using the data processing software RevMan 5.4 of the Cochrane Collaboration Network. The relative risk (RR) of the $95 \%$ confidence interval (CI) will be calculated using dichotomous variables, and the mean difference (MD) of the 95\% confidence interval $(\mathrm{Cl})$ will be calculated using continuous variables. $P<.05$ was considered statistically significant. $P$ values and $I^{2}$ values will be used to test for heterogeneity in the included study literature. $I^{2}<50 \%$ was taken as the standard for no heterogeneity or small heterogeneity, and fixed effect model was used for analysis. If heterogeneity exists or is large, $I^{2}>50 \%$ is taken as the standard, and random effect model is used for analysis. If heterogeneity exists, sensitivity analysis or subgroup analysis will be performed to determine the source of heterogeneity. Descriptive analysis will be used when meta-analysis is not possible.

Subgroup analysis: Factors such as age, course of disease, duration of treatment 
and different interventions will be analyzed by subgroup to explore the source of heterogeneity.

Sensitivity analysis: Sensitivity analysis will be used to explore the significant heterogeneity in the studies and identify the sources of heterogeneity.

Country(ies) involved: China.

Keywords: Chinese massage, acute mastitis, Systematic review and metaanalysis, protocol.

Contributions of each author:

Author 1 - Longsheng Ren.

Author 2 - Ruiying Guo.

Author 3 - Can Lv.

Author 4 - Danyang Zhao.

Author 5 - Zhihao Dong.

Author 6 - Zenglin He.

Author 7 - Qiang Wang. 